\title{
Multicolor femtosecond pump-probe system with single-electron sensitivity at low temperatures and high magnetic fields
}

Cite as: Rev. Sci. Instrum. 90, 123003 (2019); doi: 10.1063/1.5126157

Submitted: 30 August 2019 - Accepted: 30 November 2019 • Published Online: 19 December 2019
C. Traum,
P. Henzler,
S. Lohner, ${ }^{\top}$ H. Becker,
D. Nabben,' P. Gumbsheimer,'
C. Hinz

S. Mahapatra, ${ }^{2, a)}$ K. Brunner, ${ }^{2}$

D. V. Seletskiy, ${ }^{1,3}$ (D) and A. Leitenstorfer ${ }^{1, b}$

\section{AFFILIATIONS}

${ }^{1}$ Department of Physics and Center for Applied Photonics, University of Konstanz, D-78464 Konstanz, Germany

${ }^{2}$ Institute of Physics, EP3, University of Würzburg, D-97074 Würzburg, Germany

${ }^{3}$ Department of Engineering Physics, Polytechnique Montréal, Montréal, Québec H3T 1J4, Canada

a) Current address: Department of Physics, Indian Institute of Technology Bombay, Mumbai, India.

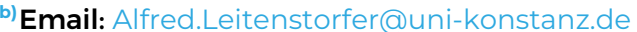

\begin{abstract}
We present an ultrafast spectroscopy system designed for temporal and spectral resolution of transient transmission changes after excitation of single electrons in solid-state quantum structures. The system is designed for optimum long-term stability, offering the option of hands-off operation over several days. Pump and probe pulses are generated in a versatile Er:fiber laser system where visible photon energies may be tuned independently from $1.90 \mathrm{eV}$ to $2.51 \mathrm{eV}$ in three parallel branches. Bandwidth-limited pulse durations between $100 \mathrm{fs}$ and 10 ps are available. The solid-state quantum systems under investigation are mounted in a closed-cycle superconducting magnet cryostat providing temperatures down to $1.6 \mathrm{~K}$ and magnetic fields of up to $9 \mathrm{~T}$. The free-standing cryomagnet is coupled to the laser system by means of a highbandwidth active beam steering unit to eliminate residual low-frequency mechanical vibrations of the pulse tube coolers. High-NA objective lenses inside the sample chamber are employed for focusing femtosecond laser pulses onto the sample and recollection of the transmission signal. The transmitted probe light is dispersed in a grating monochromator equipped with a liquid nitrogen-cooled CCD camera, enabling a frame rate of $559 \mathrm{~Hz}$. In order to eliminate spurious background effects due to low-frequency changes in the thermal equilibrium of the sample, we operate with a lock-in scheme where, instead of the pump amplitude, the pump-probe timing is modulated. This feature is provided without any mechanical action by an electro-optic timing unit inside the femtosecond Er:fiber system. The performance of the instrument is tested with spectrally resolved pump-probe measurements on a single negatively charged CdSe/ZnSe quantum dot under a magnetic field of 9 T. Selective initialization and readout of charge and spin states is carried out via two different femtosecond laser pulses. High-quality results on subpicosecond intraband relaxation dynamics after single-electron excitation motivate a broad variety of future experiments in ultrafast quantum optics and few-fermion quantum dynamics.
\end{abstract}

Published under license by AIP Publishing. https://doi.org/10.1063/1.5126157

\section{INTRODUCTION}

Derivation of the modern postulates of quantum physics has been largely completed by the first part of the 20th century, marking the first quantum revolution in science. ${ }^{1}$ Termed "second quantum revolution", it is the contemporary motivation to engineer quantum systems in order to carry out certain practical tasks, the most sought-after being quantum computation and quantum information processing. The race for the quantum computer is still in its infancy, and the question of the best candidate system is far from being settled. Regardless of the details, however, all approaches require cryogenic temperatures to combat the detrimental effects of decoherence. At this time, superconducting Josephson junctions represent the platform coming closest to real-world applications but require operational temperatures below $100 \mathrm{mK}^{2}$ and therefore a sophisticated cooling apparatus. Among other top contenders, there 
exist both atomic and solid-state approaches, with one of the latter types being the Loss-DiVincenzo quantum computer, based on single-electron quantum dot (QD) gates. ${ }^{3}$ Quantum dot (QD) approaches require less aggressive cooling strategies; however, they suffer from shorter decoherence times in comparison with, e.g., atomic-based counterparts.

In our studies, we ask the question whether certain quantum processing tasks may be carried out in solid-state systems on ultrafast time scales, thus not being limited by dephasing on picosecond to nanosecond time scales? Quantum emitters, especially those based on II-VI semiconductor heterostructures, are characterized by strong confinement potentials and large dipole moments that render them attractive platforms for studying light-matter interactions. ${ }^{4-6}$ Of special interest is the reduced screening found in these material systems. It leads to strong carrier exchange interactions that lift the degeneracy of the electronic fine structure and allow for addressing single electronic states by femtosecond laser pulses. As a result, ultrafast relaxation dynamics of distinct charge and spin configurations can be studied by femtosecond pump-probe spectroscopy. ${ }^{7-9}$ Moreover, we have already demonstrated quantum-dot based operation of a single-photon addition to a bright multimode coherent state. ${ }^{10}$ While we are currently developing strategies to extend such functionality efficiently toward few-photon coherent inputs, ${ }^{11}$ detailed investigations on single quantum systems are often challenged by weak signals potentially distorted by thermal artifacts. 8,10

Here, we present an instrumental platform specifically designed for high-precision three-color pump-probe spectroscopy on single quantum emitters inside a low-temperature magnetic cryostat. The schematic setup of the system is illustrated in Fig. 1, presenting the main components from left to right, i.e., in the same order in which they are detailed in Secs II and III of this presentation. Section II describes the highly stable three-color Er:fiber laser system designed for ultrafast selective initialization and readout of single quantum-confined electronic states. It features the implementation of a specially developed pump-probe scheme for suppression of parasitic artifacts in differential transmission signals. The closed-cycle superconducting magnetic cryostat and its spatial coupling to the laser system by means of a broadband active beam stabilization are presented in Sec. III. Along with describing the sample structure containing single negatively charged CdSe/ZnSe QDs, a comprehensive stability analysis of the magnetic cryostation and the demonstration of shot-noise limited optical detection of differential transmission signals are the subject of this section. The overall performance of the experimental system is demonstrated in Sec. IV for the case of two-color pump-probe spectroscopy on a single CdSe/ZnSe quantum dot, where ultrafast buildup of inversion in the QD

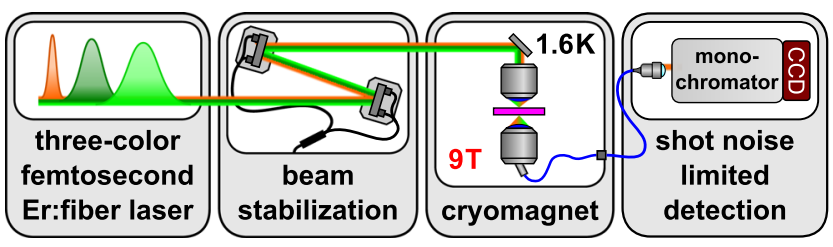

FIG. 1. Schematic of the main components of the instrumentation system, specifically developed for ultrafast three-color pump-probe spectroscopy on single quantum emitters situated inside a modern semiautomated magnetic cryostat. amplifier is time resolved while in an external magnetic field of $B=9$ T. Finally, a conclusion and outlook are given in Sec. V.

\section{LASER SYSTEM AND METHODOLOGY}

A detailed description of the Er:fiber laser and the nonlinear frequency conversion stages is given in Sec. II A. The pump-probe scheme developed for passive suppression of parasitic artifacts in transient transmission signals of single quantum systems is presented in Sec. II B. This feature requires active modulation of the pump-pulse timing at switching times faster than the interpulse distance of $25 \mathrm{~ns}$. Its technical implementation is also subject of this section.

\section{A. Highly versatile three-color femtosecond Er:fiber system}

As sketched in Fig. 2, a soliton-mode-locked Er:fiber ringoscillator $^{12}$ represents the front end of our laser system. Built entirely from polarization-maintaining single-mode fibers (PMSMFs) and passively mode-locked by a semiconductor saturable absorber mirror, it generates a highly stable near-infrared train of femtosecond pulses at a repetition rate of $f_{\text {rep }}=40 \mathrm{MHz}$. The output spectrum is centered around a photon energy of $E_{p h}=0.79 \mathrm{eV}$ and features a spectral bandwidth of $\Delta E=5.7 \mathrm{meV}$. An Er:fiber preamplifier scales the output power to $P_{\text {avg }}=10 \mathrm{~mW}$ and seeds three parallel Er:fiber amplifier branches that are precisely synchronized due to the high passive stability of the all-fiber approach. A portion of the output containing laser pulses with an energy of $25 \mathrm{pJ}$ is used to directly seed the probe branch. The remaining pulse energy seeds the two pump

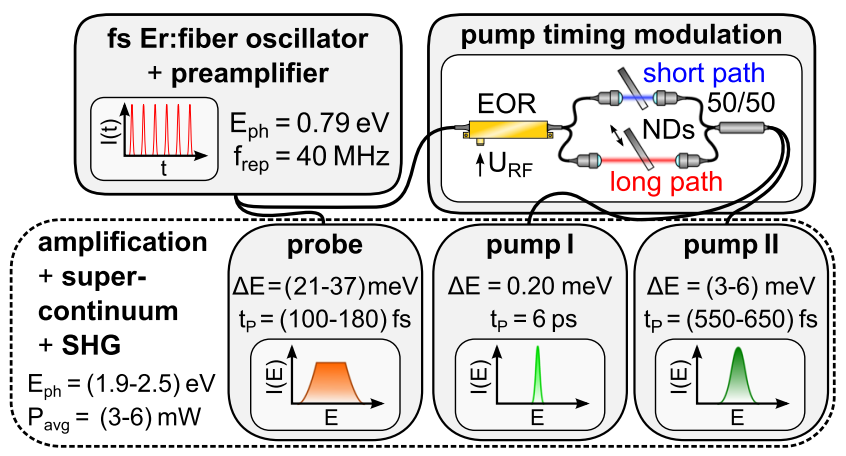

FIG. 2. Schematic of the three-color femtosecond laser system with built-in pumpprobe timing modulation. An Er:fiber oscillator followed by an Er:fiber preamplifier outputs a near-infrared pulse train at a repetition rate of $f_{\text {rep }}=40 \mathrm{MHz}$ at a center photon energy of $E_{p h}=0.79 \mathrm{eV}$. This beam seeds three synchronized laser branches (probe, pump I, and pump II). Each branch consists of a femtosecond Er:fiber amplifier with consecutive coherent supercontinuum generation in a highly nonlinear fiber. Efficient conversion into the visible range is implemented by second harmonic generation in periodically poled $\mathrm{LiNbO}_{3}$ crystals. The fan-out design of the poling period enables flexible tuning of the photon energy. The output spectra of visible laser pulses are modified in two 4f-shapers equipped with a spatial light modulator (probe) and a pair of razor blades (pump I) in their respective Fourier planes. The timing modulation is implemented by means of a free-space delay line (short and long path) controlled via a fiber-coupled electro-optical router (EOR). $U_{R F}$ denotes the driving voltage of the EOR. Neutral density filters (NDs, one adjustable) ensure identical seed power in both arms of the delay line. 
branches, preceded by a timing modulation stage, which is described in detail in Sec. II B. Each laser branch contains a nonlinear Er:fiber amplifier, providing output pulses with at least $45 \mathrm{~kW}$ peak power. This value enables efficient and particularly coherent frequency conversion $^{13}$ in highly nonlinear germanosilicate bulk fibers. Featuring a reduced core radius for an enhanced nonlinearity and a carefully designed dispersion profile, these elements are capable of generating broadband near-infrared pulses with tailor-cut output spectra. ${ }^{14}$ The high photon energy output of the dispersive branch of the supercontinuum can be optimized to cover the entire spectral range between $E_{p h}=0.86 \mathrm{eV}$ and $1.40 \mathrm{eV}$. A spectral bandwidth of up to $\Delta E$ $=540 \mathrm{meV}$ permits the generation of near-infrared few-cycle pulses with pulse durations down to $t_{P}=5.8 \mathrm{fs} .{ }^{15}$ Consecutive secondharmonic generation in periodically poled $\mathrm{LiNbO}_{3}$ crystals (PPLNs) converts the near-infrared laser light into the visible spectral range, while spectral compression due to sum frequency mixing guarantees conversion efficiencies in excess of 30\%. ${ }^{16}$ The fan-out design of the poling period of the PPLNs allows for flexible tuning of the output pulse photon energy within a spectral range between $E_{p h}=1.90 \mathrm{eV}$ and $2.51 \mathrm{eV}$. The three laser branches are equipped with different PPLN lengths to allow for generation of bandwidth-limited pulses with different durations, as required for various experiments. The probe branch generates light pulses with spectral widths between $\Delta E$ $=21 \mathrm{meV}$ and $37 \mathrm{meV}$ in order to read out a broad energy interval in transient transmission spectroscopy. As shown in Sec. III D, the sinc-shaped spectral intensity leads to a photon energy-dependent shot-noise level. This effect is avoided by transforming into a flattop spectrum by means of spectrally dependent attenuation in a spatial light modulator (SLM) situated in the Fourier plane of a $4 \mathrm{f}$ shaper. Controlled by a custom-designed self-learning algorithm, a modulated output spectrum is depicted in Fig. 8(c). The minimal temporal duration of the probe pulses is $t_{P}=100 \mathrm{fs}$. Output spectra generated in the pump I branch are as narrow as $\Delta E=(1.0$ $\pm 0.2) \mathrm{meV}$. The spectral bandwidth can be further reduced by two razor blades in the Fourier plane of a second $4 \mathrm{f}$ shaper, providing a minimal width of $\Delta E=0.2 \mathrm{meV}$ independent of central photon energy. These pulses target the selective initialization of single confined electronic states and are perfectly suited for high-resolution single-dot photoluminescence excitation (PLE) spectroscopy, as presented in Fig. 9. Representing a compromise between selective optical excitation of spectrally narrow electronic resonances in single quantum systems and maintaining a femtosecond temporal resolution in ultrafast pump-probe spectroscopy, branch pump II provides laser pulses with bandwidths ranging from $3 \mathrm{meV}$ to $6 \mathrm{meV}$. This interval corresponds to pulse durations between $t_{P}=400 \mathrm{fs}$ and $800 \mathrm{fs}$. The average output power of each of the three laser branches is larger than $P_{\text {avg }}>3 \mathrm{~mW}$ (75 pJ), which easily allows us to drive typical quantum emitters beyond the linear absorption regime. Therefore, we typically operate with substantial attenuation in ultrafast pump-probe spectroscopy on single QDs.

\section{B. Suppression of parasitic artifacts by pump-probe timing modulation}

In a typical pump-probe experiment, the spectrally resolved relative differential transmission $\Delta T / T$ of the probe pulses is measured. $\Delta T / T$ is defined as the normalized difference between the transmission through the sample previously excited by a pump pulse and the transmission through the quantum system being in its ground state. By switching between both settings with a modulation frequency $f_{\text {mod }}$ and measuring the transmitted probe spectra with, e.g., a CCD array, the spectrally resolved $\Delta T / T$ signal is detected. This setting represents a lock-in scheme for data acquisition which effectively suppresses noise contributions to $\Delta T / T$ at frequencies different to $f_{\text {mod }}$. In the standard case, amplitude modulation is performed where the pump pulses are switched off to probe the ground state transmission. Note that less than one electron is excited per pulse on average when studying individual quantum systems. Such experiments are more demanding as compared to typical ensemble measurements where a large number of molecules or an extended region of a solid-state structure is involved. While the increased sensitivity requirements are obvious, there is also a number of parasitic artifacts that are specific to working on single quantum systems. Important examples are (i) thermal shifts of sharp electronic resonances due to spurious absorption of the pump in, e.g., a metal structure surrounding the microscopic system under study and (ii) background luminescence of the sample structure. These processes are induced by the pump that is modulated at frequency $f_{\text {mod }}$, and hence, they contribute to the differential transmission signal. Nevertheless, many parasitic processes either exhibit time constants much longer than the interpulse distance that is in the nanosecond regime (e.g., thermal heating) or they are present also when the probe beam is absent (e.g., spontaneous emission of luminescence). Instead of amplitude modulation, in this work, we modulate the timing between pump and probe pulses; see Fig. 3. In one interval of the lock-in scheme, readout is taken at a positive time delay $t_{D}$ between pump and probe pulses (which may be varied to gain information on the dynamics, blue-shaded part at the left-hand side of Fig. 3). The other setting corresponds to a negative time delay where the probe precedes the pump. Here, only the residual signals that are left over from the preceding pump pulse are detected (red-shaded part in Fig. 3). ${ }^{10,17}$ Ideally, the time shift $t_{S}$ should be set to a value short compared to the interpulse distance $1 / f_{\text {rep }}$ ( $25 \mathrm{~ns}$ in our system) but long enough to make sure that the time delay in the reference phase always remains negative when varying the time delay between the pump and the probe.

We implement this strategy with a technical element in the femtosecond laser source that allows us to optoelectronically switch the

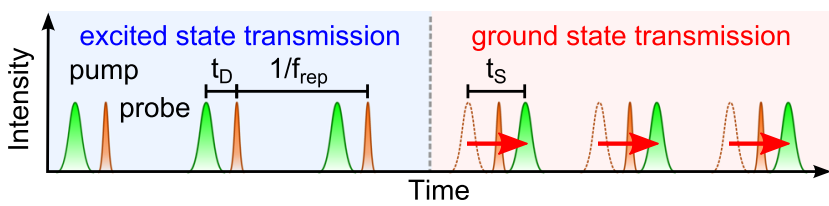

FIG. 3. Modulation scheme for pump-probe experiments, based on a temporal shift of the time delay. The first setting (left, blue color) constitutes pump and probe pulses with a normally ordered arrival sequence separated by a positive relative delay $t_{D}$, thus detecting the sample being in its excited state. In the second timing phase (right, red color), the delay of the pump pulses by the time shift $t_{S}$ after the probe pulses allows for read-out of the quantum system in a spurious state of excitation which originates from the previous excitation event in the pulse train. The interpulse distance of $25 \mathrm{~ns}$ corresponds to the inverse of the laser repetition rate of $f_{\text {rep }}=40 \mathrm{MHz}$. The dashed gray line indicates the switching between the two settings for measuring the relative differential transmission in a lock-in scheme. 
timing between pump and probe pulse trains by an interval of $t_{S}$ $=700$ ps, i.e., without any mechanical movement and at a speed faster than the interpulse distance. The scheme is depicted in the right upper corner of Fig. 2. After a beam splitter separating the pump and probe branches, a fiber-coupled electro-optic router (EOR) is located in the pump branch. Depending on the driving voltage $U_{R F}$, this element guides the incoming seed laser pulses for both pumps I and II to one of its two fiber exits (see Fig. 2). A freespace collimator is spliced to each path, and each beam is coupled back into the fiber path after a different propagation length in free space. The optical path difference between the short path (blue path in Fig. 3, left panel in Fig. 4) and the long path (red, right panel) corresponds to a pump-probe timing jump of $t_{S}=700 \mathrm{ps}$. Free space propagation is exploited here in order to ensure identical dispersion in both passes. Finally, both optical paths are recombined by means of a 50:50 fiber coupler, ensuring that the propagating laser pulses are equally distributed to the two pump branches I and II of the laser system. Figure 4(a) shows an oscilloscope trace with $400 \mathrm{MHz}$ bandwidth that is triggered by the probe branch and registers the pump pulse train. The temporal evolution of the switching voltage $U_{R F}$ of the EOR is depicted in green. For $U_{R F}=-2.8 \mathrm{~V}$, the pump pulses travel along the short path of the free-space delay line (blue). When switching the driving voltage to $U_{R F}=2.8 \mathrm{~V}$, the long path is selected (red) where the pump-probe timing is increased by $t_{S}=700 \mathrm{ps}$. The EOR bandwidth of $3.4 \mathrm{GHz}$ permits the pulse-by-pulse modulation of the pump arm and therefore operation up to the Nyquist limit. To obtain clean measurements, the properties of the pump pulses have to be as independent as possible from the choice of the delay line path they propagate through. Therefore, the fiber lengths of both paths are adjusted to be exactly the same. Furthermore, two variable neutral density (ND) filters located in both free-space paths compensate for any difference in optical losses, balancing the intensity transmitted through the delay unit with a high degree of precision. Figure 4(b) compares the spectral densities of the visible pump pulses in absolute units between the output of the short path (blue) and the long path (red). The maximum of the absolute spectral difference (green) amounts to $37 \mu \mathrm{W} / \mathrm{THz}$, which corresponds to a relative spectral deviation of $1.4 \%$ between the spectra.
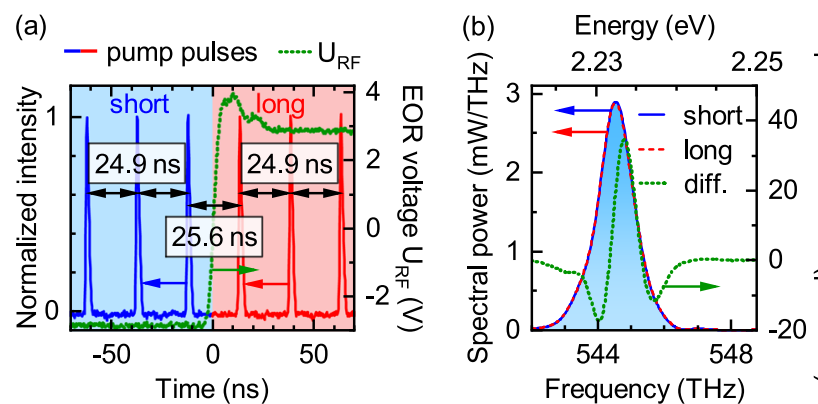

FIG. 4. (a) Visible pump pulse trains as detected with a Si photodiode by an oscilloscope triggered to the probe pulses. Short (blue) and long path (red) pulse trains are delayed with respect to each other by the action of the EOR. When the switching voltage $U_{R F}$ (green) is set from $-2.8 \mathrm{~V}$ to $2.8 \mathrm{~V}$, the pump pulses experience an abrupt time shift of $t_{S}=700 \mathrm{ps}$. (b) Spectra of pump pulses propagating along short and long paths of the delay line. The residual difference of both spectra is plotted in green.
Note that the repetition rate of our laser system could be easily reduced by placing a fiber-coupled electro-optic modulator, e.g., after the preamplifier. ${ }^{12}$ In this way, also quantum systems returning to their ground state on relatively long time scales beyond several nanoseconds could be studied in detail.

\section{LOW-TEMPERATURE MAGNETIC CRYOSTAT, SAMPLES, AND SIGNAL DETECTION}

The second major component of our scientific instrument is the closed-cycle superconducting magnetic cryostat. Section III A describes the coupling of pump and probe pulses to the cryomagnet and the spectrally resolved detection of transmitted probe pulses. Typical sample structures containing single CdSe/ZnSe quantum dots are presented in Sec. III B. A comprehensive stability analysis of the cryostation is carried out in Sec. III C, and the shot-noise-limited analysis of differential transmission is covered by Sec. III D.

\section{A. Optical-to-cryomagnet interface and signal detection}

The closed-cycle magnetic cryostat is capable of virtually uninterrupted operation and allows for a lowest achievable sample temperature of $T_{L}=1.6 \mathrm{~K}$. Its superconducting Helmholtz coil is able to generate a magnetic field of up to $B=9 \mathrm{~T}$ in Faraday geometry. To isolate low-frequency mechanical vibrations generated in the helium compressor of the pulse tube cooler, the cryostation is decoupled from the optical table. Therefore, a beam steering unit is mandatory to precisely stabilize the beam pointing of the femtosecond laser system relative to the cryostation. Figure 5 illustrates the experimental setup: The time delays $t_{D 1}$ and $t_{D 2}$ between the two

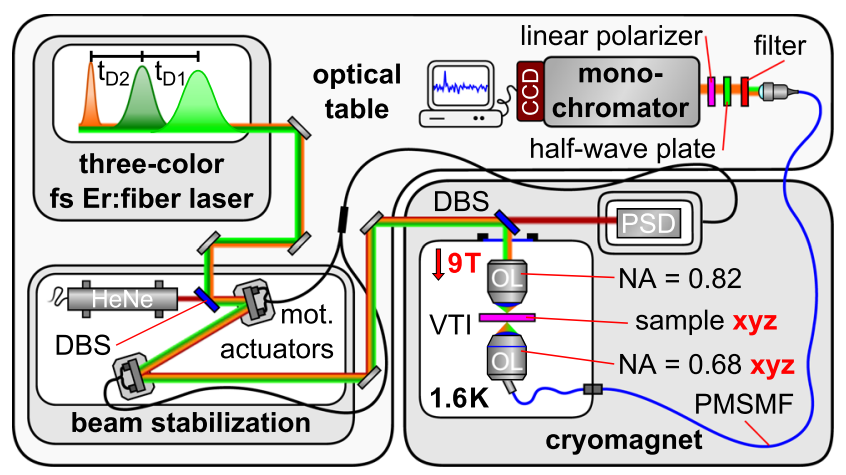

FIG. 5. Optical-to-cryomagnet interface: the beam pointing of the fiber laser into the cryostation is compensated by an active beam stabilization. Attached to the cryomagnet, a position sensitive detector (PSD) tracks the position and beam pointing of a HeNe guide laser, which is spatially overlapped with pump and probe branches of the Er:fiber laser system via dichroic beam splitters (DBSs). Pointing is stabilized by two motorized actuators. The laser pulses are focused onto the sample using an objective lens $(\mathrm{OL})$ with $N A=0.82$. A fiber-coupled $\mathrm{OL}$ with $\mathrm{NA}=0.68$ is used for collection and focusing of the probe pulses transmitted through the sample structure into a polarization-maintaining single-mode fiber (PMSMF). The sample and collection objective are aligned in all three directions by means of linear piezopositioners. The fiber guides the transmitted probe signal to the optical table. The spectrally resolved differential transmission signal is obtained in a grating monochromator equipped with a liquid $\mathrm{N}_{2}$-cooled CCD camera. 
pump (light green and dark green) and probe pulse trains (orange) are implemented by means of computer-controlled linear mechanical delay stages, located in the optical path of the probe and one pump branch (not shown). A first dichroic beam splitter (DBS) spatially overlaps the femtosecond laser pulses with a HeNe guide laser (red). All four beams are then directed toward the cryostation by two piezo-driven actuators attached to the optical table. Located in ambient conditions on top of the cryomagnet, a second DBS separates the guide laser from pump and probe beams, sending the former into a position-sensitive detector (PSD). Tracking the beam pointing of the guide laser with two quadrant photodiodes, it generates an error signal processed by a proportional-integral-derivative controller of a bandwidth of $8 \mathrm{kHz}$. The signal is fed back to the motorized actuators that compensate for any drifts in the beam pointing of pump and probe pulses in the cryostation by stabilizing the error signals for the beam position and pointing direction detected with the PSD. The femtosecond laser pulses are reflected into the transmission microscope situated in the variable temperature insert (VTI). A low-temperature objective lens (OL) with NA $=0.82$ focuses the pump and probe beams onto the sample structure. Light transmitted through the sample structure is collected by a fiber-coupled lowtemperature objective lens with $\mathrm{NA}=0.68$. Both sample and collection objective may be aligned in $\mathrm{x}, \mathrm{y}$, and $\mathrm{z}$ directions by means of linear closed-loop piezopositioners with nanometer resolution. The transmitted laser pulses are guided back to the optical table by a polarization-maintaining single-mode fiber (PMSMF). After blocking the pump by a dichroic filter with a $>10^{5}$ contrast, the probe pulses are dispersed in an aberration-minimized grating spectrometer. 1340 spectral channels are detected by a liquid nitrogen-cooled CCD camera at a spectral resolution of $100 \mu \mathrm{eV}$, a quantum efficiency of $95 \%$, and a $1760 \mathrm{~Hz}$ frame readout rate. The polarization state of the transmitted light is detected with a combination of linear polarizer and half-wave plate, both located in front of the entrance slit of the spectrograph.

\section{B. Single CdSe/ZnSe quantum dots embedded into nanophotonic structures}

In order to demonstrate the performance of our system, we investigate $\mathrm{CdSe} / \mathrm{ZnSe}$ QDs embedded into nanophotonic structures to maximize the interaction with transmitted probe photons. As illustrated in Fig. 6(a), single QDs are formed by self-assembled CdSe islands (red cones), which show diameters between $3 \mathrm{~nm}$ and $6 \mathrm{~nm}$ with heights ranging from $2 \mathrm{~nm}$ to $4 \mathrm{~nm} .{ }^{18}$ They are enclosed

(a)

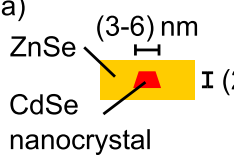

(b)

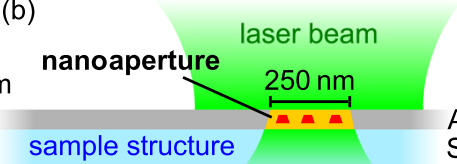

into an epitaxially grown ZnSe matrix of $100 \mathrm{~nm}$ thickness. This epilayer is removed from the original substrate by a lift-off process and optically contacted to a $120 \mu \mathrm{m}$-thick crystalline quartz $\left(\mathrm{SiO}_{2}\right)$ substrate. $\mathrm{A} \mathrm{Ga}^{+}$-based focused ion beam is used to pattern the epilayer into $\mathrm{ZnSe}$ discs (yellow), which subsequently are embedded into aluminum (Al, dark gray) by means of a sequence of evaporation and etching steps. ${ }^{6}$ As sketched in Fig. 6(b), the resulting structure represents a nanoaperture containing single QDs. Its diameter of $250 \mathrm{~nm}$ is adjusted to exploit the extraordinary transmission properties of these subwavelength structures. Together with reducing the effective beam radius of the incident laser light, this feature enhances the light-matter interaction. ${ }^{19}$ Furthermore, the nanoapertures are fabricated with nearly perfect circular shape (not shown), rendering them applicable for polarization-sensitive measurements. Each structure contains on average 10 QDs, whose electronic resonances are spectrally separable due to the intrinsic size dispersion of the selfassembled CdSe islands. This fact allows for optical addressing of single QDs.

\section{Stability analysis of the magnetic cryostation}

The mechanical stability of the cryostation is quantified by detecting the probe transmission through an aluminum nanoaperture of a diameter of $250 \mathrm{~nm}$. The sample temperature is set to $T_{L}$ $=1.6 \mathrm{~K}$. The sampling frequency of the transmission signal is set to $4 \mathrm{kHz}$. To characterize the influence of (i) the active beam stabilization and (ii) the helium compressor on the probe transmission, we switch on or off one or both of the components. The upper graph of Fig. 7(a) (black) shows the transmission signal when both components are switched off. Its relative standard deviation amounts to
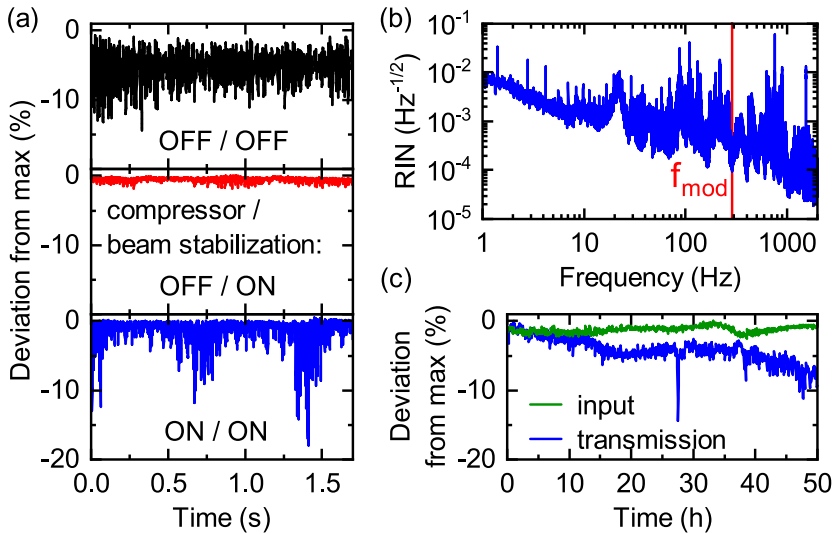

FIG. 7. (a) Analysis of the average probe power transmitted through a nanoaperture with a diameter of $250 \mathrm{~nm}$ sitting at a temperature of $T_{L}=1.6 \mathrm{~K}$ inside the cryostation with a readout rate of $4 \mathrm{kHz}$. In the upper graph, the He compressor and beam stabilization are switched off (black). Upon activation of the beam stabilization, the noise amplitude reduces drastically (central graph, red). Switching on the compressor induces acoustic noise, detected as a periodic modulation of the transmitted power (lower graph, blue). (b) Relative amplitude noise spectrum of the transmitted signal when the He compressor and beam stabilization are both operating. The modulation frequency $f_{\text {mod }}=279.3 \mathrm{~Hz}$ selected for the experiments is marked by a vertical red line. (c) Relative deviation of probe power over a time period of $50 \mathrm{~h}$ (blue). The temporal evolution of the average input power is shown in green. 
2.3\%. Upon activation of the high-bandwidth active beam steering (center graph, red), the relative standard deviation of the transmission reduces by nearly a factor of 8 to a value of $0.3 \%$. Switching on the He compressor induces low-frequency disturbances. Originating from the pressure buildup in the pulse tubes installed next to the VTI of the cryomagnet, this motion cannot be compensated by the active beam stabilization. The mechanical vibrations translate into a periodic modulation of the transmitted power [lower graph in Fig. 7(a), blue] where the relative amplitude of most fluctuations remains at or below $5 \%$ of the maximum signal. The operation frequency of the pulsed tube at $1.4 \mathrm{~Hz}$ and its higher harmonics are clearly discernible in Fig. 7(b), where the relative intensity noise (RIN) of the transmitted signal is shown when the compressor and beam stabilization are both switched on. Spectrally broad peaks between $20 \mathrm{~Hz}$ and $800 \mathrm{~Hz}$ are due to the mechanical resonance frequencies of the optical transmission microscope assembly, held at the bore of the cryomagnet by arms of a length of approximately $1 \mathrm{~m}$. Despite these features, the overall shape of the RIN signal exhibits a hyperbolic frequency dependence, thus providing a clear motivation for high-frequency readout. In our implementation, the modulation frequency $f_{\text {mod }}$ is carefully set to $279.3 \mathrm{~Hz}$ (red vertical line) to avoid higher harmonics of the operation frequency visible throughout the entire spectral range. Furthermore, this frequency represents the optimal compromise between the noise amplitude of the transmitted signal and the relative read-out noise of the CCD camera. To underline the option for long-term hands-free operation of our system, the transmission signal is depicted over a time interval of $50 \mathrm{~h}$ as a blue graph in Fig. 7(c). The excellent stability of the average input laser power is shown in green. This analysis proves that uninterrupted operation without any adjustment of our system is possible over several days, thus enabling an unprecedented signal-to-noise ratio in nonlinear and ultrafast studies on single-electron quantum systems.

\section{Shot-noise-limited detection of spectrally resolved differential transmission}

The noise properties of the probe pulse train transmitted through the nanoaperture are now analyzed with the spectrometer and the CCD camera. We record the intensity in two spectral channels A (green) and B (violet) normalized by the respective mean transmission $T_{\text {mean }}$ at a frame rate of $f_{\text {meas }}=558.6 \mathrm{~Hz}$ over a time interval of $142 \mathrm{~s}$ [left-hand side of Fig. 8(a)]. Both pixels are separated by an energy interval of $3.3 \mathrm{meV}$ that is larger than the spectral width of the expected differential transmission features induced by the quantum system [compare Fig. 8(b)]. The spectral positions of channels A and B are marked in Fig. 8(c). The noise in both channels is dominated by the amplitude fluctuations induced by the acoustic vibrations of the cryosystem. For comparison, the relative intensity difference of both time traces $\mathrm{A}-\mathrm{B}$ is plotted in blue in Fig. 8(a). A histogram of the likelihood for specific readout values A-B is given in light blue at the right-hand side of Fig. 8(a). It turns out that the width of a Gaussian normal distribution $\sigma_{\Sigma}$ (solid blue line) fitted to these data is virtually identical to the distribution $\sigma_{S}$ (dashed red) expected when assuming a Poissonian statistics of the difference of the photoelectron number detected in each readout event. Obviously, the signal A-B is limited by shot noise only. This finding demonstrates a high degree of correlation of the technical
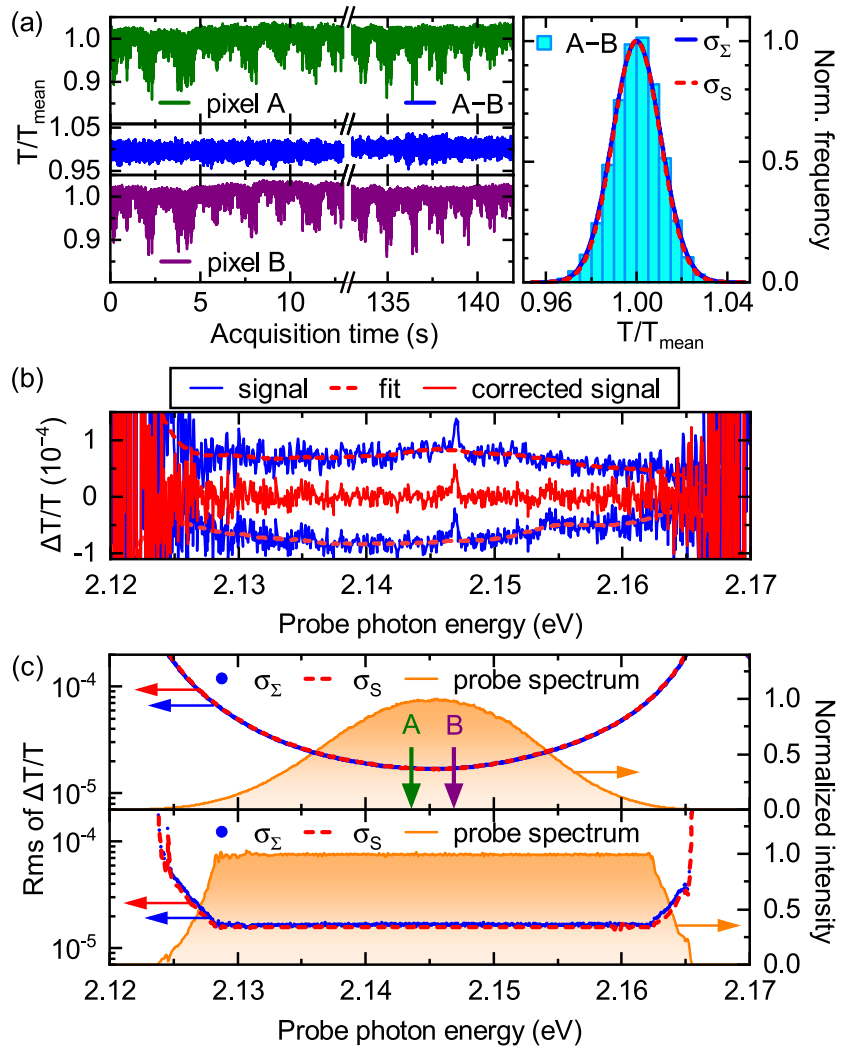

FIG. 8. (a) Left-hand side: Time traces of probe spectral intensities, acquired in CCD channels A (green) and B (violet) at a frame rate of $f_{\text {meas }}=558.6 \mathrm{~Hz}$. The channels are separated in energy by $3.3 \mathrm{meV}$, and their spectral positions are marked in the upper graph of (c). The relative difference of both channels $A-B$ is plotted in blue. The corresponding signal histogram is depicted in light blue at the right-hand side. The width of a fitted Gaussian normal distribution ( $\sigma_{\Sigma}$, solid blue) originates almost entirely from fundamental shot noise $\left(\sigma_{S}\right.$, dashed red), underlining the strong correlation of acoustic noise in intensity traces A and B. (b) Two relative differential transmission spectra obtained under nominally identical integration conditions are depicted as solid blue lines. $4 \times 10^{4}$ individual probe pulse spectra were detected at a modulation frequency of $f_{\text {mod }}=279.3 \mathrm{~Hz}$, resulting in an integration time of $142 \mathrm{~s}$, respectively, and the time delay $t_{D}$ was set to $100 \mathrm{ps}$. At a photon energy of $2.147 \mathrm{eV}$, a spectrally narrow feature can be assigned to the stimulated emission from the QD. By subtraction of a spline interpolation (red dashed line), the spectrally broad background originating from acoustic noise induced by the cryostation is removed from the data (red solid line). (c) Root-mean-square of spectrally resolved relative differential transmission (blue dots), entirely dictated by fundamental shot noise (red dashed line). The spectral intensity of probe pulses (orange with shading, upper graph) leads to an energy-dependent noise floor with a minimum value of $1.6 \times 10^{-5}$. Transforming the probe pulse spectrum into a flattop by means of pulse shaping with an SLM (see Sec. II A) results in an energy independent noise floor within an energy interval of $34 \mathrm{meV}$ (lower graph).

noise in the transmission signals A and B: the transverse mechanical vibrations of the nanoaperture with respect to the confocal position of the probe are modulating the transmitted spectrum only in its total amplitude with negligible deviations between different frequency positions. Therefore, the vibration-induced distortions of the transmitted probe spectra may be clearly distinguished from the spectrally narrowband signals from the quantum system we want 
to detect. Figure 8(b) shows two relative differential transmission spectra as blue lines that were taken under nominally identical conditions. Each dataset is averaged over $4 \times 10^{4}$ readouts of the CCD that were acquired at a modulation frequency of $f_{\text {mod }}=279.3 \mathrm{~Hz}$. The time delay between pump and probe pulses is set to $t_{D}=100 \mathrm{ps}$. At a probe photon energy of $2.147 \mathrm{eV}$, a narrowband spectral feature emerges. It originates from stimulated emission of single photons by the quantum system. ${ }^{8}$ Due to the strong correlated noise contributed by acoustic vibrations, the signature is situated on top of a positive or negative background, which varies owing to the strong but correlated contribution due to the technical vibrations. Therefore, we perform a least-square fit to the broadband background based on a spline interpolation of third order (dashed red line) and subtract it from the data. The resulting background-corrected relative differential transmission spectra are centered around zero in the regions where no specific signal features occur. An example is shown as a solid red line in Fig. 8(b). Implemented in the experiment as real-time procession of acquired $\Delta T / T$-spectra, this procedure establishes a balanced detection of the narrowband changes in the quantum system in the differential transmission that is limited only by shot noise. The upper graph of Fig. 8(c) depicts the calculated root-mean-square value (rms) of the relative differential transmission signal (blue dots), resolved for all pixels on the CCD sensor. The integration time of $142 \mathrm{~s}$ leads to a noise floor of $1.6 \times 10^{-5}$ in the center of the spectrum where the photon flux per channel is maximum. The theoretical shot noise limit is indicated by the red dots, taking into account the spectral shape of the transmitted fJ-probe pulse (orange with shading). It turns out that our signals are shot-noise-limited over the entire spectral range of the probe after subtraction of the correlated acoustic noise. The pronounced variation of the spectral intensity of the transmitted probe pulses leads to a strong dependence of the noise floor. In experiments on single-electron systems, however, only a fraction of the power available from our femtosecond laser source may be used for probing in order to remain in a linear limit. This fact allows us to actively transform the spectral intensity of the probe pulses into a flat-top distribution via shaping the intensity amplitude in a $4 \mathrm{f}$ configuration containing an SLM (see Sec. II A). The resulting spectrum is presented as an orange graph with shading in the lower graph of Fig. 8(c). As expected, the corresponding noise level remains constant within a wide interval of photon energy covering $34 \mathrm{meV}$. On the other side, the constant flux of probe power also allows us to operate at the optimum level of input intensity for all spectral channels that is set by the saturation level of the CCD array per readout event. In this way, we ensure that the maximum noise performance is reached over the entire spectral width covered by the probe pulses.

\section{ULTRAFAST PUMP-PROBE SPECTROSCOPY ON SINGLE CdSe/ZnSe QUANTUM DOTS}

The sensitivity of our experimental setup is demonstrated in a two-color pump-probe experiment on a single CdSe/ZnSe QD in a magnetic field of $9 \mathrm{~T}$. To this end, the precise knowledge of the electronic structure of the QD outlined in Sec. IV A is a crucial prerequisite. Consecutively, the femtosecond dynamics of two photo-excited charge and spin configurations are described in Sec. IV B.

\section{A. Optical characterization of the electronic structure}

Photoluminescence (PL) and photoluminescence excitation (PLE) spectra of a single negatively charged CdSe/ZnSe QD in a magnetic field of $B=9 \mathrm{~T}$ in Faraday geometry may be found in Fig. 9 . The sample temperature is set to $T_{L}=1.6 \mathrm{~K}$. The QD is optically excited by $\sigma^{+}$(i.e., right-handed) circularly polarized laser pulses at an average power of $P_{e x c}=3 \mu \mathrm{W}$. The red solid line depicts the PL spectrum of the QD under a pump photon energy of $E_{p u}=2.278 \mathrm{eV}$. Centered around an energy of $2.1817 \mathrm{eV}$, two Zeeman components of the fundamental trion emission $\mathrm{X}^{-}$are separated by $0.8 \mathrm{meV}$. As illustrated in the gray box, $\mathrm{X}^{-}$represents the radiative recombination of the trion ground state $| \pm 3 / 2\rangle_{0}$ (XGS), consisting of two electrons and one hole in the lowest energy shells in the conduction (s) and valence (S) bands, respectively. The twofold energy degeneracy is lifted by the external magnetic field. We denote a specific quantum state of the trion as $\left|\mathrm{F}_{z}\right\rangle_{S}=\left|\mathrm{S}^{2}, \mathrm{~S}_{z}\right\rangle\left|\mathrm{j}_{z}\right\rangle . \mathrm{F}=\mathrm{S}+\mathrm{j}$ is the overall angular momentum, consisting of the total electron spin $S$ $=\mathrm{s}_{1}+\mathrm{s}_{2}$ and the hole spin $\mathrm{j} . \mathrm{S}_{z}$ and $\mathrm{j}_{z}$ are projections along the $\mathbf{z}$ axis, respectively, with $\mathbf{z} \| \mathbf{B}$. After recombination of the photoexcited electron-hole pair, the QD returns to its Zeeman-split ground state consisting of one single electron in its s-shell $| \pm 1 / 2\rangle$. Consequently, for the XGS where $S=0$, the PL emission consists of a line doublet with a low-energy component $X_{\sigma^{-}}^{-}$and its high-energy counterpart $X_{\sigma^{+}}^{-}$that exhibit left- and right-handed circular polarization, respectively. ${ }^{20}$ Redshifted by an energy of $4.7 \mathrm{meV}$, a weaker doublet originates from radiative recombination of charged biexciton ground states into excited trion triplet states. ${ }^{21}$ These resonances are not discussed in the present work. The PLE spectrum recorded by integrating over the entire $\mathrm{X}^{-}$emission and varying the pump photon energy

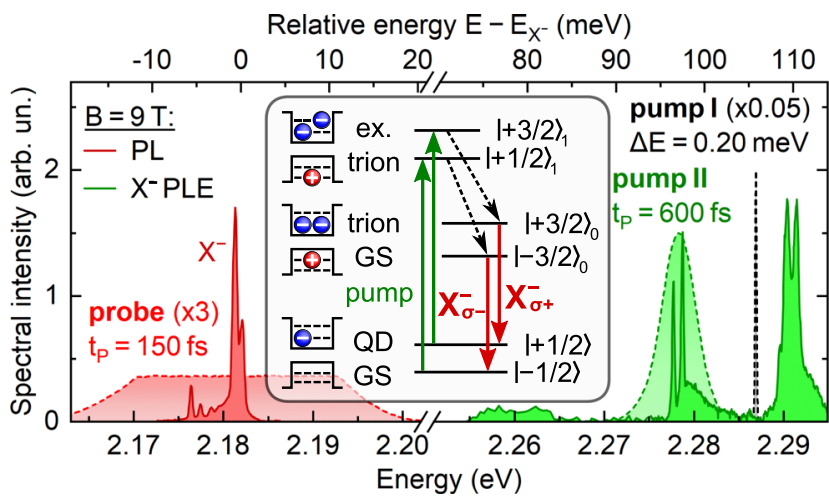

FIG. 9. Stationary characterization of a single negatively charged CdSe/ZnSe quantum dot (QD) in a magnetic field of $B=9 \mathrm{~T}$ and at a lattice temperature of $T_{L}=1.6 \mathrm{~K}$. The red solid line shows the photoluminescence $(\mathrm{PL})$ of a single $\mathrm{QD}$, centered at $E_{X_{-}}=2.18 \mathrm{eV}$. The twofold degeneracy of the fundamental trion resonance $\mathrm{X}^{-}$is lifted by the magnetic field. A photoluminescence excitation spectrum ( $X^{-}-P L E$, green solid line) of energy resolution $0.2 \mathrm{meV}$ reveals optical transitions at higher energy $\left(E-E_{X-}\right)$. These measurements are performed using pump pulses generated in the pump I branch of the laser system with an average power of $P_{p u}=3 \mu \mathrm{W}$. An example spectrum is represented by the black dashed graph. Spectral intensities of the probe and pump II pulses used for the ultrafast two-color pump-probe experiments are shown in red and green dashed lines with shading, respectively. The inset graph qualitatively sketches excited and read-out optical transitions of the $\mathrm{QD}$. 
is depicted in solid green. The energy resolution of only $200 \mu \mathrm{eV}$ is given by the spectrally narrowband excitation pulse generated in the pump I branch (example spectrum given as a black dashed graph). This measurement was obtained in a fully automated way by motorized scanning of both the poling period of the fan-out PPLN crystal used for second harmonic generation (SHG) (coarse adjustment of wavelength) and the angle of the diffraction grating in the 4f-shaper (fine spectral selection, see Sec. II A). The PLE trace shows three main absorption features. We concentrate on the line doublet centered around an energy of $2.278 \mathrm{eV}$. These resonances are identified as optical transitions of the QD from its ground state into the triplet state of the excited trion, consisting of a charge configuration where two electrons are in the p-shell and s-shell, respectively, and one hole resides in the P-shell. ${ }^{10,22} \sigma^{+}$-polarized pump photons are coupling to two of the four excited trion triplet states, namely, $|+1 / 2\rangle_{1}$ and $|+3 / 2\rangle_{1}$ (see the gray box). The corresponding optical transitions are separated by $1.0 \mathrm{meV}$ due to a combined action of electron-hole exchange (EHX) and magnetic field. ${ }^{21,23}$

\section{B. Femtosecond few-fermion dynamics in a strong magnetic field}

The central photon energy of the $\sigma^{+}$-polarized pump pulses is now tuned to $2.278 \mathrm{eV}$. Owing to the pulse duration of $t_{p u}=600 \mathrm{fs}$, the spectral bandwidth covers both excited trion triplet states $|+1 / 2\rangle_{1}$ and $|+3 / 2\rangle_{1}$ (see the dashed green line with shading in Fig. 9). The average excitation power is set to $P_{p u}=3 \mu \mathrm{W}$. The photon energy of the linearly polarized probe is tuned to $2.180 \mathrm{eV}$, simultaneously coupling to the low- and high-energy Zeeman components of the fundamental trion resonance (red dashed line with shading in Fig. 9). The average probe power is set to $P_{p r}=1 \mu \mathrm{W}$ at a pulse duration of $t_{p r}=100 \mathrm{fs}$. An external magnetic field of $B=9 \mathrm{~T}$ in Faraday geometry is applied, and the sample is cooled down to a temperature of $T_{L}=1.6 \mathrm{~K}$. Figure 10(a) depicts the color-coded spectrally resolved differential transmission $\Delta T / T$ of a single QD for pumpprobe time delays $t_{D}$ between $-50 \mathrm{ps}$ and $300 \mathrm{ps}$. $\Delta T / T$ spectra for representative time delays are given in detail in Figs. 10(b)-10(e). For large negative delays $t_{D} \ll 0$ ps where the probe pulses precede the pump pulses, no pump-induced changes are expected in the signal. This is directly reflected in Fig. 10(b), underscoring the absence of any long-lived parasitic (e.g., thermally induced) artifacts due to the timing modulation explained in Sec. II B. For $-5 \mathrm{ps}<t_{D}<0 \mathrm{ps}$, a delay-dependent frequency modulation centered around the lowenergy component of $\mathrm{X}^{-}$is caused by the perturbed dephasing of the probe-induced polarization. The effect is successfully captured by the numerical solution of dynamical Bloch equations for a two-level system [orange solid line in Fig. 10(c)]. ${ }^{8,10}$ For positive time delays, we observe a striking feature with two spectral components $X_{\sigma^{-}}^{-}$and $X_{\sigma^{+}}^{-}$at energies of the Zeeman components of $\mathrm{X}^{-}$. The corresponding PL spectrum is depicted in Fig. 10(b) as a red dashed line. The positive differential transmission originates from two microscopic effects: (i) Coulomb interactions between photoexcited carriers shift electronic resonances of the quantum system quasi-instantaneously, resulting in a steep increase in signal amplitude on a time scale of less than 1 ps. (ii) Intraband scattering of hot carriers leads to relaxation toward the XGS. This state represents an inverted system providing optical gain limited to stimulated emission of a single photon that may be triggered by the probe pulse. As illustrated in
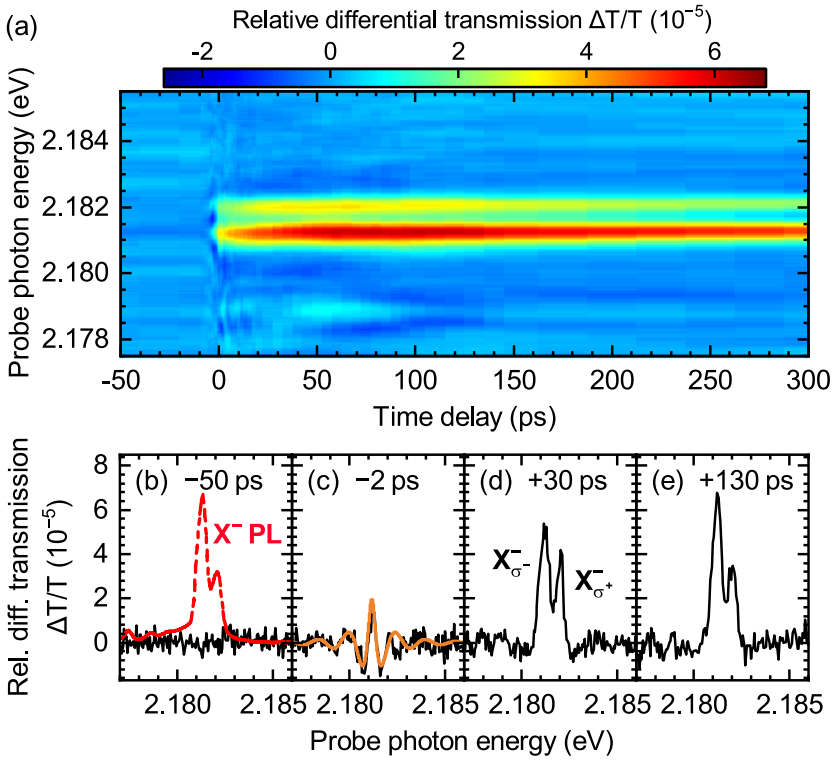

(f)

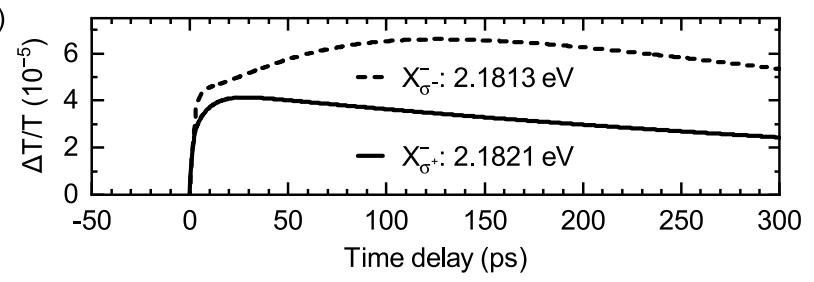

FIG. 10. (a) Spectrally resolved differential transmission $\Delta T / T$ of a single negatively charged $\mathrm{CdSe} / \mathrm{ZnSe} \mathrm{QD}$ as a function of time delay $t_{\mathrm{D}}$ between pump and probe pulses. The magnetic field strength is set to $B=9 \mathrm{~T}$, and the sample temperature is $T_{L}=1.6 \mathrm{~K}$. The photon energy of the $\sigma^{+}$polarized pump pulses is tuned to excite trion triplet states in the $\mathrm{QD}$ with the average power of $\mathrm{P}_{\mathrm{pu}}=3 \mu \mathrm{W}$. Probe pulses are linearly polarized with an average power of $\mathrm{P}_{\mathrm{pr}}=1 \mu \mathrm{W}$, and the photon energy is centered on the fundamental trion resonance $X^{-}$. (b) $\Delta T / T$ spectrum for $t_{D}=-50 \mathrm{ps}$. The red dashed graph shows the $X^{-} P L$ of the $Q D$. (c) $\Delta T / T$ spectrum for $t_{D}=-2 \mathrm{ps}$. The spectral modulation is due to a perturbed free induction decay. A model fit based on the numerical solution of two-level optical Bloch equations is depicted in orange. (d) and (e) show $\Delta T / T$ spectra for $t_{D}=30 \mathrm{ps}$ and $130 \mathrm{ps}$, where single photon gain of low- and high-energy Zeeman-components $\mathbf{X}_{\sigma^{-}}^{-}$and $\mathbf{X}_{\sigma^{+}}^{-}$is maximal, respectively. For positive time delays, fits for $\Delta T / T$ signals $X_{\sigma^{-}}^{-}$ and $\mathbf{X}_{\boldsymbol{\sigma}^{+}}^{-}$obtained from rate equations are given in (f) as black solid and dashed lines.

Fig. 9, the dynamics of the low-energy component $X_{\sigma^{-}}^{-}$at a photon energy of $2.1813 \mathrm{eV}$ reflects the carrier relaxation from excited trion triplet state $|+1 / 2\rangle_{1}$, whereas the signal $X_{\sigma^{+}}^{-}$at an energy of $2.1821 \mathrm{eV}$ monitors the relaxation of the state $|+3 / 2\rangle_{1} \cdot{ }^{10,22,24}$ By comparing the amplitudes of both signals in $\Delta T / T$ spectra for $t_{D}=30 \mathrm{ps}$ and $130 \mathrm{ps}$ presented in Figs. 10(d) and 10(e), a markedly different relaxation dynamics of the triplet states toward the XGS is evident. First, the relaxation of the excited trion triplet state $|+3 / 2\rangle_{1}$ is discussed. Its relaxation toward the singlet-type XGS with a total angular momentum projection of $\mathrm{F}_{z}=+3 / 2$ requires a change in total electron spin from 1 to 0 , which might be a relatively slow process. ${ }^{24,25}$ In QDs with an elongated confinement potential, however, the anisotropic electron-electron exchange (EEX) mixes triplet and singlet states, 
resulting in a finite overlap of the triplet state $|+3 / 2\rangle_{1}$ with the singlet state $|+3 / 2\rangle_{0}$ of the excited trion. ${ }^{26,27}$ This fact opens up a direct relaxation channel of the excited trion triplet state into the XGS via the emission of acoustic or optical phonons. ${ }^{24}$ As a result, the dynamics of the high-energy component $X_{\sigma^{+}}^{-}$can be described in a three-level system. ${ }^{10}$ The time constant $\tau_{3 / 2}$ is associated with the nonradiative relaxation of $|+3 / 2\rangle_{1}$, therefore increasing the population of the XGS. Its population decreases exponentially with a lifetime $\tau_{s}$, accounting for the spontaneous recombination of the trion. The solution of the corresponding rate equation for the signal amplitude of $X_{\sigma^{+}}^{-}$is depicted in Fig. $10(\mathrm{f})$ as a solid black line. Full inversion of the quantum state is achieved after $\tau_{3 / 2}=(8 \pm 2) \mathrm{ps}$, giving rise to maximum single photon gain. The spontaneous lifetime is extracted to be $\tau_{s}=(500 \pm 45) \mathrm{ps}$. The low-energy signal $X_{\sigma^{-}}^{-}$exhibits a distinctly different temporal evolution, with full single photon gain established only after approximately $130 \mathrm{ps}$. This finding directly reflects the slow relaxation of the excited trion triplet state $|+1 / 2\rangle_{1}$. The consecutive relaxation toward the XGS with $F_{z}=-3 / 2$ requires both a change in the total electron spin $\Delta S=-1$ and total angular momentum projection $\Delta \mathrm{F}_{z}=-2$. Whereas the change in total electron spin is governed by the singlet-triplet mixture as discussed for $X_{\sigma^{+}}^{-}$, the change in total spin is provided by an admixture of the triplet states $|+3 / 2\rangle_{1}$ to the state $|+1 / 2\rangle_{1}$ mediated by the anisotropic EHX. ${ }^{10,21,24}$ In a microscopic picture, this process corresponds to a simultaneous spin flip of electron and hole that is associated with an additional time constant $\tau_{1 / 2}$. Therefore, a four-level system has to be used for describing the relaxation of the $|+1 / 2\rangle_{1}$ state. The resulting dynamics calculated by the rate equation for the lowenergy component $X_{\sigma^{-}}^{-}$is given as a black dashed line. The time it takes for a combined electron-hole spin flip is measured to be $\tau_{1 / 2}$ $=(70 \pm 12)$ ps. We stress that the entire map presented in Fig. 10(a) was acquired completely in a hands-off operation of the instrument. In combination with other benchmark tests performed in Secs. III D and IV A, we believe these results showcase our setup as a remarkable tool for analyzing and manipulating interactions of ultrafast wave packets with few-particle excitations in single quantum systems.

\section{CONCLUSION AND OUTLOOK}

We present a multicolor pump-probe setup for time- and energy-resolved detection of transient transmission changes in single quantum structures. Major features defining the novelty of our setup are as follows: (i) A highly versatile Er:fiber laser system provides three precisely synchronized pulse trains. The duration of the bandwidth-limited output pulses may be adjusted between 100 fs and $10 \mathrm{ps}$, and the central photon energy may be independently tuned over a large part of the visible range. A minimum output bandwidth of $0.2 \mathrm{meV}$ allows for selective initialization of single electronic states in high-resolution photoluminescence excitation spectroscopy. A broadband probe spectrum with flat-top shape provides a maximum dynamic range in single-electron differential transmission. (ii) Detection of transmission changes is based on an all-optical pump-probe timing modulation. This feature enables efficient suppression of parasitic artifacts such as thermal shifts of narrowband electronic resonances or background luminescence. (iii) A closedcycle magnet cryostat enables experiments at temperatures down to $1.6 \mathrm{~K}$ and in external magnetic fields up to $9 \mathrm{~T}$ at virtually unin- terrupted operation. The mechanical vibrations inherent to its pulse tube coolers necessitate decoupling from the optical table. An active beam steering unit is employed to stabilize the pointing of pump and probe beams into the cryostation. A comprehensive stability analysis of the complete experimental setup is performed. Owing to the combination of extreme sensitivity with excellent long-term stability and robustness, we believe that our instrument represents an advanced platform for analyzing and manipulating interactions of ultrafast wave packets with few-particle excitations in individual quantum structures. This claim is supported by performing two-color pumpprobe spectroscopy revealing the ultrafast buildup of inversion in the trion ground state of a negatively charged $\mathrm{CdSe} / \mathrm{ZnSe}$ quantum dot. Acquired over $56 \mathrm{~h}$ of continuous hands-off operation, the time- and spectrally resolved transient transmission data are free of parasitic artifacts at an excellent signal-to-noise ratio.

In future experiments, we intend to exploit the full potential of our setup in three-color two-dimensional spectroscopy. By using pairs of femtosecond pump pulses, correlation effects between confined electronic states become directly accessible. We expect such studies to deliver unprecedented insight into the elementary dynamics of not only conventional semiconductor quantum dots but also novel solid-state quantum systems, e.g., defect centers in widegap materials or laterally confined states in two-dimensional matrices such as transition metal dichalcogenide monolayers. In this way, vital information may be collected toward future applications of such systems in advanced quantum technologies enabling, e.g., the transfer of data at a superior level of security and with minimum consumption of energy.

\section{ACKNOWLEDGMENTS}

Funding by DFG via SFB767 (Project No. B02) and Grant No. INST 38/521-1 is gratefully acknowledged.

\section{REFERENCES}

${ }^{1}$ P. Michler, Quantum Dots for Quantum Information Technologies (Springer, 2017).

${ }^{2}$ M. H. Devoret and R. J. Schoelkopf, Science 339, 1169 (2013).

${ }^{3}$ A. Imamoglu, D. D. Awschalom, G. Burkard, D. P. DiVincenzo, D. Loss, M. Sherwin, and A. Small, Phys. Rev. Lett. 83, 4204 (1999).

${ }^{4}$ D. Press, T. D. Ladd, B. Zhang, and Y. Yamamoto, Nature 456, 218 (2008).

${ }^{5}$ M. Bayer, G. Ortner, O. Stern, A. Kuther, A. A. Gorbunov, A. Forchel, P. Hawrylak, S. Fafard, K. Hinzer, T. L. Reinecke, S. N. Walck, J. P. Reithmaier, F. Klopf, and F. Schäfer, Phys. Rev. B 65, 195315 (2002).

${ }^{6}$ A. Zavatta, S. Viciani, and M. Bellini, Science 306, 660 (2004).

${ }^{7}$ D. Gammon and D. G. Steel, Phys. Today 55(10), 36 (2002).

${ }^{8}$ F. Sotier, T. Thomay, T. Hanke, J. Korger, S. Mahapatra, A. Frey, K. Brunner, R. Bratschitsch, and A. Leitenstorfer, Nat. Phys. 5, 352 (2009).

${ }^{9}$ U. Woggon, Optical Properties of Semiconductor Quantum Dots (Springer, 2014).

${ }^{10}$ C. Hinz, P. Gumbsheimer, C. Traum, M. Holtkemper, B. Bauer, J. Haase, S. Mahapatra, A. Frey, K. Brunner, D. E. Reiter, T. Kuhn, D. V. Seletskiy, and A. Leitenstorfer, Phys. Rev. B 97, 045302 (2018).

${ }^{11}$ F. Werschler, B. Lindner, C. Hinz, F. Conradt, P. Gumbsheimer, Y. Behovits, C. Negele, T. de Roo, O. Tzang, S. Mecking, A. Leitenstorfer, and D. V. Seletskiy, Nano Lett. 18, 5396 (2018).

${ }^{12}$ D. Brida, G. Krauss, A. Sell, and A. Leitenstorfer, Laser Photonics Rev. 8, 409 (2014).

${ }^{13}$ S. Kumkar, G. Krauss, M. Wunram, D. Fehrenbacher, U. Demirbas, D. Brida, and A. Leitenstorfer, Opt. Lett. 37, 554 (2012). 
${ }^{14}$ A. Sell, G. Krauss, R. Scheu, R. Huber, and A. Leitenstorfer, Opt. Express 17, 1070 (2009).

${ }^{15}$ S. Baumann, W. Paul, T. Choi, C. P. Lutz, A. Ardavan, and A. J. Heinrich, Science 350, 417 (2015).

${ }^{16}$ K. Moutzouris, F. Adler, F. Sotier, D. Träutlein, and A. Leitenstorfer, Opt. Lett. 31, 1148 (2006).

${ }^{17}$ H. Shigekawa, O. Takeuchi, and M. Aoyama, Sci. Technol. Adv. Mater. 6, 582 (2005).

${ }^{18}$ S. Mahapatra, K. Brunner, and C. Bougerol, Appl. Phys. Lett. 91, 153110 (2007).

${ }^{19} \mathrm{C}$. Genet and T. W. Ebbesen, Nature 445, 39 (2007).

${ }^{20}$ I. A. Akimov, T. Flissikowski, A. Hundt, and F. Henneberger, Phys. Status Solidi 201, 412 (2004).
${ }^{21}$ I. A. Akimov, K. V. Kavokin, A. Hundt, and F. Henneberger, Phys. Rev. B 71, 075326 (2005).

${ }^{22}$ J. Huneke, I. D'Amico, P. Machnikowski, T. Thomay, R. Bratschitsch, A. Leitenstorfer, and T. Kuhn, Phys. Rev. B 84, 115320 (2011).

${ }^{23}$ K. V. Kavokin, Phys. Status Solidi 195, 592 (2003).

${ }^{24}$ M. E. Ware, E. A. Stinaff, D. Gammon, M. F. Doty, A. S. Bracker, D. Gershoni, V. L. Korenev, Ş. C. Bădescu, Y. Lyanda-Geller, and T. L. Reinecke, Phys. Rev. Lett. 95, 177403 (2005).

${ }^{25}$ Ş. C. Bădescu, Y. B. Lyanda-Geller, and T. L. Reinecke, Phys. Rev. B 72, 161304 (2005).

${ }^{26}$ K. V. Kavokin, Phys. Rev. B 69, 075302 (2004).

${ }^{27}$ M. M. Glazov and V. D. Kulakovskii, Phys. Rev. B 79, 195305 (2009). 